Reinhard Altenhöner

\title{
Auf dem Weg zu einem nationalen Zeitungsportal. Eine materialspezifische Kooperation als Treiber eines neuen Dienstes für Wissenschaft und Forschung
}

„Zeitungsdigitalisierung ist eine große Herausforderung, aber auch eine große Chance.“1

\section{Einleitung}

Als der amerikanische Konzern Google Inc. 2008 seine zwei Jahre zuvor gestartete Initiative für die Onlineaufbereitung historischer Zeitungen und das bereits früher begonnene Buchprojekt um eine dezidierte Scan-Offensive erweiterte, die kooperativ mit den Verlegern von Zeitungen erfolgen sollte, war noch davon die Rede, dass dies erst der Anfang sei: Man werde Milliarden gedruckter Zeitungsseiten aus der ganzen Welt durchsuchbar, leicht auffindbar und online zugänglich machen. ${ }^{2}$ Bereits drei Jahre später war der Enthusiasmus verflogen und Google stellte das Programm - entnervt auch durch die Auseinandersetzungen mit Verlegern $\mathrm{zu}$ urheberrechtlichen Fragestellungen - wieder ein, verwies allerdings auf die noch existierenden Projekte mit Bibliotheken. ${ }^{3}$ Ausgangspunkt für Google war die Feststellung, dass Zeitungen ein zentraler Spiegel des Zeitgeschehens über Jahrhunderte seien; das Problem bestehe darin, dass der überwiegende Teil der Zeitungen nicht online verfügbar sei, dies

1 Bürger, Thomas: Zeitungsdigitalisierung als Herausforderung und Chance für Wissenschaft und Kultur. In: Zeitschrift für Bibliothekswesen und Bibliographie 63 (2016) H. 3. S. 123-132, hier S. 132. http://dx.doi.org/10.3196/186429501663332 (28.11.2017).

2 Vgl. https://googleblog.blogspot.de/2008/09/bringing-history-online-one-newspaper.html (28.11.2017).

3 Vgl. https://www.heise.de/newsticker/meldung/Google-stoppt-Digitalisierung-von-Zeitungen-1246655.html Der ursprüngliche Blogeintrag (http://thephoenix.com/BLOGS/phlog/archive/2011/05/19/google-abandons-master-plan-to-archive-the-world-s-newspapers.aspx) ist im Netz nicht mehr erreichbar und auch nicht archiviert. Hinweis auf das LoC-Projekt unter https://comlaw.wordpress.com/2011/06/06/the-phlog-google-abandons-master-plan-to-archive-the-worlds-newspapers/. Immerhin rund 2000 Zeitungen, überwiegend aus dem angloamerikanischen Raum, werden von Google noch vorgehalten: https://news.google.com/newspapers, der eigene Sucheinstieg ist allerdings abgeschaltet, vgl. http://news.google.com/archivesearch (alle Zugriffe am 28.11.2017).

ว Open Access. (C) 2018 Reinhard Altenhöner, publiziert von De Gruyter. (G) BY-Nc-ND Dieses Werk ist lizenziert unter der Creative Commons Attribution-NonCommercial-NoDerivatives 4.0 Lizenz. 
wolle man ändern. ${ }^{4}$ Diese Sicht wird in zunehmendem Maße von der interessierten Öffentlichkeit, von Vertretern aus Wissenschaft und Forschung, aber ebenso von den bestandshaltenden Einrichtungen wie Bibliotheken und Archiven geteilt. $^{5}$

Erfolgreicher war das Google-Programm mit seinem Buch-Projekt, ${ }^{6}$ innerhalb dessen auch in nennenswertem Umfang Zeitungen digitalisiert wurde. Beim deutschen Partner, der Bayerischen Staatsbibliothek (BSB) wurde so nahezu der gesamte urheberrechtsfreie Zeitungsbestand mit über 1000 Titeln digitalisiert und steht seit diesem Jahr Schritt für Schritt in einer eigenen Rechercheplattform zur Verfügung. ${ }^{7}$ Gerade angesichts dieser Aktivitäten wurde deutlich, dass die Digitalisierung historischer Zeitungen in Deutschland im internationalen Vergleich eine geringe Rolle spielt. ${ }^{8}$

Inzwischen zeichnet sich ab, dass die Deutsche Forschungsgemeinschaft (DFG) im Frühjahr 2018 die seit vielen Jahren ausgesetzte Digitalisierung historischer Zeitungen wieder in die Förderung aufnehmen wird. ${ }^{9}$ Damit kommen die Bemühungen einer Reihe von Bibliotheken und ganz besonders die Anstrengungen, die Thomas Bürger über viele Jahre hinweg unternommen hat, zu einem (vorläufigen) Abschluss: Ein systematischer Ansatz zur flächigen Digitalisierung von historischen Zeitungen auch in Deutschland steht nun kurz vor seinem Start. Damit eröffnet sich die große Chance, weitere nennenswerte Volumina dieses für verschiedene Bereiche der Forschung wichtigen und oft nur

\section{Wie Anm. 2.}

5 Zuletzt mit breiterer Ausrichtung unter Hinweis auf die Forderung nach einem nationalen Zeitungsmuseum vgl. André, Michael: Zeitungen sind ein nationaler Pflegefall (4.9.2017). http://www.getidan.de/gesellschaft/michael_andre/76996/zeitungen-sind-ein-nationaler-pflegefall (30.11.2017). Vgl. auch Saam, Alena: Tagungsbericht „Presse als historische Quelle“. Tagung des Landschaftsverbands Rheinland am 13.9.2016 in Bonn. In: H-Soz-Kult, 9.11.2016. https://www.hsozkult.de/conferencereport/id/tagungsberichte-6798 (3.12.2017). Bei diesem Zusammentreffen von Wissenschaftlern, Bibliothekaren und Archivaren, das aus Anlass des von der Universitäts- und Landesbibliothek Bonn initiierten Projekts zur Digitalisierung der rheinischen Presse des 18. bis 20. Jahrhunderts stattfand, wurde anhand einer Vielzahl von Beiträgen und Werkstattberichten der besondere Quellenwert der Zeitung einerseits als Berichterstattung, mehr aber noch als Reflektor herausgestellt.

6 Vgl. dazu zusammenfassend Altenhöner, Reinhard u. a.: Digitalisierung von Kulturgut. In: Praxishandbuch Bibliotheksmanagement. Hrsg. von Rolf Griebel, Hildegard Schäffler u. Konstanze Söllner. Berlin, Boston: de Gruyter 2015. S. 763-811.

7 https://digipress.digitale-sammlungen.de/ (28.11.2017).

8 Vgl. dazu ausführlich Bürger, Zeitungsdigitalisierung (wie Anm. 1), S. 126-127.

9 Vgl. das aktuelle Merkblatt „Erschließung und Digitalisierung“ mit Stand Juli 2016, in dem auf die Ergebnisse der Pilotprojekte verwiesen wird: http://www.dfg.de/formulare/12_15/ 12_15_de.pdf, S. 4 (3.12.2017). Die Ankündigung der geplanten neuen Förderinitiative erfolgte mündlich im November 2017 auf der Halbjahrestagung der dbv-Sektion 4 in Regensburg. 
schwer zugänglichen Materials nicht nur komfortabel bereitzustellen, sondern durch Konversions- und Transformationsschritte außerdem maschinennutzbare Korpora für die Wissenschaft zu erzeugen und damit neue Nutzungsszenarien zu schaffen. Neben der Digitalisierung selbst sind dazu weitere Schritte zum Ausbau der Infrastruktur erforderlich.

Dieser Beitrag versucht, den jetzt erreichten Stand zu reflektieren, beschreibt die Rahmenbedingungen und konzentriert sich auf die Dokumentation der Vorbereitungen für den praktischen Start der neuen Förderlinie. Zugleich versucht er sich abschließend an einer Kontextualisierung der verschiedenen Anstrengungen in den Rahmen der Forschungsinfrastruktur für die Geistes-, Sozial- und Kulturwissenschaften hinein. Er stellt insofern eine Fortschreibung dessen dar, was Thomas Bürger als Fundament bereitet hat - und ist so auch eine Dankesbezeugung an ihn.

\section{Ausgangssituation}

Insbesondere im deutschen Sprachgebiet, in dem Zeitungen ${ }^{10}$ bereits früh aufkamen und eine besonders hohe Dichte an Titeln entstand, stellt dieses Material einen herausragenden, dabei aber schwer zu greifenden Bestand dar. Bestände sind oftmals nicht an einem Ort lückenlos vorhanden, das Handling ist aufgrund der Formate schwierig, die Mengen an Seiten kaum beherrschbar. ${ }^{11} \mathrm{Zu}-$ gleich ist das Material - da oftmals als „Verbrauchsware“ auf billigem Papier gedruckt - in hohem Maße vom Papierzerfall bedroht. Diese Hürden machen deutlich, dass systematische und lokale Aktivitäten zwar inzwischen nennenswerte Teile der Drucke des 16. bis 18. Jahrhunderts in die Digitalisierung genommen haben, Zeitungen hingegen immer nur in vergleichsweise kleinen Größenordnungen digitalisiert wurden. Obschon Mikroverfilmungsprogramme seit den 1960er Jahren Material in beträchtlichem Umfang gesichert haben, ist die Benutzbarkeit schwierig und sind die Ergebnisse solcher Projekte insbesondere

10 „Zeitungen“ sind aufgrund häufiger Titeländerungen, Übernahmen, Aufspaltungen usw. schwierig abgrenzbar und damit zugleich terminologisch etwas unscharf. Neben den historischen Begrifflichkeiten werden hier auch zeitungsähnliche Journale einbezogen.

11 Vgl. auch hierzu ausführlich Bürger, Zeitungsdigitalisierung (wie Anm. 1), S. 123-125. Wegweisend bereits Bürger, Thomas: Die Digitalisierung der kulturellen und wissenschaftlichen Überlieferung - Versuch einer Zwischenbilanz. In: Zeitschrift für Bibliothekswesen und Bibliographie 58 (2011) H. 3-4. S. 133-141, hier S. 138-139. http://dx.doi.org/10.3196/ 1864295011583445 (28.11.2017). 
für eine Nachbearbeitung oftmals von zweifelhafter Qualität. ${ }^{12}$ Dennoch kann auf diesen Grundlagen, soweit dies qualitativ vertretbar ist, aufgesetzt werden.

Die geschilderten Beobachtungen für die Bestände deutscher Bibliotheken verwundern umso mehr, als es in internationaler Perspektive inzwischen eine ganze Reihe von Initiativen gibt, die Digitalisate in erheblichem Umfang bereitstellen und darüber hinaus einen Schwerpunkt auf die maschinenlesbare Bereitstellung des Inhalts für Suche und Analyse legen. Vor diesem Hintergrund startete 2013 das von der DFG geförderte Projekt „Digitalisierung historischer Zeitungen" mit einer Laufzeit von zwei Jahren. Hauptziel des Projekts war es, einen systematischen und hinsichtlich der Vorgehensweise, der eingesetzten Verfahren und standardisierten Ergebnisse umfassend koordinierten Ansatz zu entwickeln. Ein besonderes Augenmerk lag dabei auf der Ermittlung der Anforderungen an Auswahl, Abfolge und Präsentationsqualität durch Forschung und Wissenschaft. Um von vornherein eine pragmatische Ausrichtung sicherzustellen, sollten verschiedene Werkzeuge, Verfahren und Erschließungstiefen in einer Reihe von Pilotprojekten unter dem Dach des Hauptprojekts erprobt werden, die Nachweis- und Präsentationsstrukturen konkret verbessert und schließlich Empfehlungen zur Auswahl unter inhaltlichen, rechtlichen, konservatorischen und organisatorischen Kriterien erarbeitet werden. Die Ergebnisse der von insgesamt sechs Bibliotheken in Bremen, Berlin, Dresden, Halle, Frankfurt am Main und München durchgeführten Pilotprojekte wurden in einem Masterplan für einen systematischen Ausbau der Zeitungsdigitalisierung zusammengetragen, der als Leitlinie für die weitere Ausgestaltung der angedachten Förderinitiative ausgelegt war. ${ }^{13}$

Damit ist klar, dass das Projekt sowohl auf praktische Aspekte der Durchführung eines großvolumigen Digitalisierungsprogrammes an vielen Standorten abzielte, zugleich aber auch die erforderlichen Module für die Koordinierung, die Aufbereitung und Bereitstellung des entstehenden Datenmaterials und den Aufbau von Zugangsstrukturen in den Blick nahm, die den Anforderungen aus Wissenschaft und Forschung adäquat sein sollten.

12 Vgl. Bürger, Zeitungsdigitalisierung (wie Anm. 1), S. 124-125.

13 Unter Federführung der Sächsischen Landesbibliothek - Staats- und Universitätsbibliothek Dresden (SLUB) arbeiteten die Staatsbibliothek zu Berlin - Preußischer Kulturbesitz (SBB), die Staats- und Universitätsbibliothek Bremen (SuUB), die Deutsche Nationalbibliothek Frankfurt am Main (DNB), die Universität- und Landesbibliothek Sachsen-Anhalt Halle (ULB) und die Bayerische Staatsbibliothek München (BSB). Vgl. http://gepris.dfg.de/gepris/projekt/ 271857751 (3.12.2017). 


\section{Projektergebnisse: Infrastrukturmodule, Pilotprojekte, Masterplan}

Das Projekt bestand aus einer Reihe von unterschiedlichen Pilotprojekten unter dem Dach des Gesamtvorhabens: So arbeiteten die DNB und die SBB an der funktionalen Verbesserung der Zeitschriftendatenbank (ZDB) als Nachweis- und Steuerungsinstrument für die Zeitungsdigitalisierung, während die SLUB den DFG-Viewer medientypologisch auf den Materialtyp Zeitung ausbaute, um eine homogene digitale Präsentation von Zeitungen zu erreichen. Eine Aktivität der SBB bezog sich auf die Weiterentwicklung der den Digitalisierungsworkflow unterstützenden Software. Alle Bibliotheken mit Ausnahme der DNB führten schließlich Projekte zur Digitalisierung ausgewählter Zeitungen durch, in denen - abhängig von den gewählten Zeitschnitten und Materialarten innerhalb des Medientypus Zeitungen - unterschiedliche Verfahren und Werkzeuge sowie verschiedene Erschließungstiefen erprobt wurden, um aufgrund der daraus ableitbaren Erkenntnisse abgestimmte Festlegungen für weiterführende Maßnahmen zu erarbeiten.

Einschließlich erforderlicher Nacharbeiten, die sich zum Teil über den Projektzeitraum hinauszogen, wurden folgende Bausteine und Quasistandards zur Vorbereitung der erforderlichen Infrastruktur erreicht:

1 Für die ZDB wurde zur Verbesserung der Nutzerorientierung und Funktionalität und unter Nutzung der Onlinedatenschnittstelle der ZDB eine neue Präsentationsoberfläche geschaffen, die Wissenschaftlerinnen und Wissenschaftlern transparenter als zuvor differenzierte Quelleninformationen und Bestandsnachweise für den Materialtyp Zeitung bietet (z. B. durch die Visualisierung des relationalen Umfelds eines ausgewählten Titels) und den zeitungsbesitzenden Institutionen als Datenplattform für Zeitungsdigitalisierungsprojekte dienen kann. ${ }^{14}$

2 Die SLUB realisierte eine Erweiterung des DFG-Viewers um medientypologisch erforderliche spezifische Zugangspunkte und Nutzungsmerkmale (Kalenderfunktion, Zoom, Panning, verteilte Volltextsuche). Dazu wurden einheitliche Mindeststandards für die Präsentation von Zeitungen erarbeitet, die einen einheitlichen Zugang zu aggregierten projektspezifischen Prä-

14 Die neue Präsentationsschicht ist erreichbar unter http://zdb-katalog.de/index.xhtml (28.11.2017). Vgl. auch Hubrich, Jessica u. Hans-Jörg Lieder: Zeitungssuche interaktiv - Der neue ZDB-Webkatalog. In: o-bib 1 (2014) H. 1. S. 305-311. https://www.o-bib.de/article/view/ 2014H1S305-311 (3.12.2017). Ferner: Hubrich, Jessica u. Hans-Jörg Lieder: Die Zeitschriftendatenbank und die Digitalisierung historischer Zeitungen in Deutschland. In: Dialog mit Bibliotheken 28 (2016) H. 2. S. 22-28. 
sentationen erlauben. Zugleich entstand so eine angepasste Ausbaustufe des bekannten kostenfrei nachnutzbaren Präsentationstools für entsprechende Initiativen auf kommunaler Ebene. ${ }^{15}$

3 Das Workflow-Tool Goobi zur Unterstützung von Digitalisierungsprojekten wurde zielgerichtet erweitert; die Ergebnisse sind in die Open-SourceSoftware Kitodo integriert.

4 URN Granular: Die ULB Halle erprobte und implementierte die persistente fragmentbasierte Adressierung und Referenzierung (URN Granular 2.0) in Kooperation mit der DNB mit dem Ziel, künftig Artikel und Seiten persistent adressieren zu können. ${ }^{16}$

5 Optical Character Recognition (OCR) brachte insgesamt verwertbare Ergebnisse, auch wenn die Probleme beispielsweise mit Überschriften in sehr unterschiedlichen Schriftarten, Schriftstilen oder Schriftmischungen eine weitere Optimierung der Werkzeuge erforderlich machen. Vor diesem Hintergrund wird der Anschluss an das von der DFG geförderte „Koordinierungsprojekt zur Weiterentwicklung von Verfahren der Optical Character Recognition (OCR-D)“17 gesucht, das die Verfahren der automatischen Texterkennung und Konzepte für optimale Workflows näher analysieren und verbessern will. Zugleich entstand ein Set von Qualitätsanforderungen, mit dessen Hilfe nicht nur eine optimale Benutzung sichergestellt werden soll, sondern ebenso die maschinelle Weiternutzung der entstehenden Daten möglich wird. Im Wesentlichen konnten hier auch für den Medientyp Zeitungen die bestehenden Empfehlungen der DFG bestätigt werden bzw. sind dort schon eingegangen. ${ }^{18}$

Neben der koordinierten Weiterentwicklung/Auswertung wurden in den Pilotprojekten zur Digitalisierung unterschiedliche Verfahren und Vorgehensmodelle zum Ablauf erprobt und damit digitale Zeitungsseiten im Umfang von 1,5 Millionen erzeugt.

15 Vgl. Bürger, Zeitungsdigitalisierung (wie Anm. 1), S. 129.

16 Vgl. Sommer, Dorothea u. a.: Zeitungsdigitalisierung: eine neue Herausforderung für die ULB Halle. Werkstattbericht aus der Pilotphase des DFG-Projekts „Digitalisierung historischer Zeitungen“. In: ABI Technik 34 (2014) H. 2. S. 75-85. doi.org/10.1515/abitech-2014-0013 (3.12.2017).

17 http://www.ocr-d.de/ (28.11.2017).

18 Deutsche Forschungsgemeinschaft: Praxisregeln „Digitalisierung“. Bonn 2016. http://www. dfg.de/formulare/12_151/12_151_de.pdf (3.12.2017). Vgl. auch Dietsche, Anne, Alain Dubois u. Florian Steffen: Standards Zeitungsdigitalisierung, 2014. https://www.digicoord.ch/images/0/ 08/Standards_Zeitungsdigitalisierung_DE_20140709.pdf (3.12.2017). 
- Die SuUB digitalisierte ihren über Jahrzehnte gesammelten Bestand von Reproduktionen deutschsprachiger Zeitungen des 17. Jahrhunderts (ca. 500 Zeitungsunternehmen mit 750 Titeln und 375000 Seiten). ${ }^{19}$

- Die SLUB erprobte Verfahren der Massendigitalisierung anhand sechs verfilmter Zeitungen und die Digitalisierung einer Zeitung vom Original mit exemplarischer Erprobung bibliothekarischer Artikelerschließung einschließlich GND-Verknüpfung der Artikelautoren.

- Die ULB digitalisierte mit dem Hallischen Tageblatt eine überregional bedeutsame Zeitung des Pietismus vom Original, erprobte OCR-Verfahren und die vertiefte Strukturdatenerfassung bis auf die Artikelebene.

- Die BSB bearbeitete zwei überregional gewichtige Zeitungen mit unterschiedlichen Schwerpunkten: Neben der dienstleistergestützten Massendigitalisierung wurden OCR, Strukturierungs- und Artikelseparierungsarbeiten - zum Teil halbautomatisch - durchgeführt und ausgewertet.

Weitere Themen über alle Pilotprojekte hinweg waren die Evaluierung und Schärfung der Qualitätsanforderungen insbesondere für eine maschinelle Nachverarbeitung, unter denen vom Film oder Original digitalisiert werden sollte, sowie die Ermittlung der Aufwände für verschiedene Aspekte der Tiefenerschließung.

Im Ergebnis hat das Projekt hierzu ein Stufenmodell entwickelt, das abhängig vom Material und den Anforderungen insgesamt sechs, aufeinander aufbauende Stufen einer Grund- und Tiefenerschließung (in zwei Erweiterungsebenen) abbildet, denen unterschiedliche Anwendungsszenarien und Recherchebedarfe zugeordnet werden. Das Projekt empfiehlt, alle Zeitungen mit dem Grundstandard Stufe 1 (DFG-Viewer-Strukturdatenset) und Stufe 2 (OCR-Erkennung) zu erschließen. Bei den weitergehenden Stufen (halbautomatisch/mit manuellen Eingriffen, Artikelsegmentierung, Verknüpfung zu Normdaten sowie weiteren Erschließungsmaßnahmen bis hin zur Bilderkennung) stoßen die etablierten Verfahren technisch an Grenzen, sodass hier weitere Impulse erforderlich sind (s. u.). Nach Möglichkeit sollte eine halbautomatische Erschließung insbesonde-

19 Hermes, Maria u. Maria Elisabeth Müller: Digitalisierung der vollständigen deutschsprachigen Zeitungen des 17. Jahrhunderts an der SuUB Bremen - Ein Werkstattbericht. In: o-bib 1 (2014) H. 1. S. 265-279, hier S. 274. https://www.o-bib.de/article/view/2014H1S265-279/1168 (3.12.2017). Aufgrund der materialspezifischen Situation war hier OCR ebenso wie Optical Layout Recognition (OLR) unmöglich. Vgl. auch in leicht aktualisierter Fassung: Müller, Maria Elisabeth u. Maria Hermes-Wladarsch: Die Digitalisierung der deutschsprachigen Zeitungen des 17. Jahrhunderts - ein Projekt mit Komplexität! In: Die Zeitung als Medium in der neueren Sprachgeschichte. Korpora - Analyse - Wirkung. Hrsg. von Oliver Pfefferkorn, Joerg Riecke u. Britt-Marie Schuster. Berlin, Boston: de Gruyter 2017. S. 39-59. 
re bei standardisierten, gleichförmigen Titelverläufen erfolgen. Die Layouterkennung und die Artikelseparierung haben dabei für die wissenschaftliche Arbeit mit dem Material eine hohe Relevanz und sind insbesondere bei Zeitungen von überregionaler Bedeutung (Leitmedien, innovative Zeitungen, fachspezifisch relevante Zeitungen und Journale) zu empfehlen.

Der aufgrund der verschiedenen Erfahrungen und Ergebnisse formulierte Masterplan mit Empfehlungen für eine Hauptphase „Digitalisierung historischer Zeitungen in Deutschland“ wurde der DFG Anfang 2016 in einer ersten Fassung vorgelegt. Im Rahmen der sich anschließenden Evaluierung dieser Empfehlungen durch die Gutachter der DFG und weiterer Arbeiten im Konsortium reifte der Masterplan nun unter Federführung der SBB zu einer zweiten Version heran; es entstanden ihn begleitende Werkzeuge und Checklisten, die insgesamt die Basis für eine konkrete Vorplanung der künftigen Förderinitiative bilden. Die Ergebnisse wurden im Sommer 2017 der DFG vorgelegt.

\section{Der Masterplan: Leitplanken für die neue Förderinitiative der DFG}

\section{Anforderungen der Wissenschaft}

Zentrale Elemente für jedwede Initiative sind die Auswahl des zu digitalisierenden Materials einerseits und die erwarteten Ergebnisse andererseits. Diese Merkmale wurden im Projekt im Blick auf andere Projekte und durch Workshops mit Wissenschaftlerinnen und Wissenschaftlern erarbeitet. Im Vordergrund stand die Forderung, möglichst schnell zu beginnen und möglichst viel Material bereitzustellen - auch unter Inkaufnahme qualitativer Abstriche; dabei sollten grundsätzlich der ganze geografische Raum und die ganze zeitliche Erstreckung angemessen repräsentiert sein. Darüber hinaus sind die unterschiedlichen Anforderungen und Fragestellungen der verschiedenen Wissenschaftsdisziplinen zu berücksichtigen. So ist aus der Perspektive der historisch forschenden Fachdisziplinen für Big-Data-Analysen und andere Methoden der Digital Humanities die Bereitstellung von unter Umständen auch aufbereiteten Volltexten von Belang, für andere Disziplinen stellt die Kalendersuche als $\mathrm{Zu}$ gang zu den Images ein ausreichendes Angebot dar. Von allen Beteiligten wurde die Durchführung von OCR als wünschenswert erachtet.

Im Ergebnis liegt ein Set von Empfehlungen vor, das große Zentren (Orte), „Dauerbrenner“, Leitmedien, Innovatoren, aber auch thematische Kollektionen, 
historisch bedeutsame Zeitungen, das politische Spektrum ebenso wie regionale Zentren mit Beispielen unterlegt benennt und einen guten Ansatzpunkt für die weitere Auswahl oder Festlegung bietet. Zugleich wird schon hier deutlich, dass die pure Erzeugung von Images von Zeitungsseiten nicht ausreicht, sondern ergänzende Arbeiten im Bereich der Erschließung und der Infrastruktur hinzukommen. ${ }^{20}$

\section{Inhaltlicher Fokus, Mengen und Kostenaspekte, Koordination und Steuerung}

Im Rahmen des Projekts vorgenommene Ermittlungen in der ZDB ergaben 21583 Zeitungstitel aus dem Zeitraum 1600-1945, die in deutscher Sprache gedruckt oder im deutschen Sprachraum erschienen sind. ${ }^{21}$ Für rund 4000 davon sind Digitalisate angegeben, häufig aber noch nicht zugänglich erschlossen (oder als Projekt noch in der Planung bzw. Bearbeitung), außerdem handelt es sich nicht selten bloß um Teilbestände. Die Bestände verteilen sich auf 1654 besitzende Einrichtungen, vorwiegend Bibliotheken, da Archivbestände (mit Ausnahme Bayerns) noch nicht systematisch aufgenommen wurden. Rund ein Dutzend Bibliotheken weist mehr als 1000 Titel nach, nur rund 40 mehr als 500 . Trotz aller Unsicherheiten, die bei diesen Angaben bestehen, wird doch deutlich, dass ein umfassender Ansatz zur Digitalisierung von einer großen Zahl zu beteiligender Einrichtungen ausgehen muss. Hinzu kommt, dass Bestände einer einzigen Zeitung oftmals auf mehrere besitzende Einrichtungen verteilt aufbewahrt werden, sodass eine die gesamte Laufzeit eines Titels berücksichtigende Digitalisierung häufig nur als Kooperationsprojekt mehrerer Einrichtungen sinnvoll ist. Auch wenn also z. B. der größte Bestand in Deutschland in der SBB rund $30 \%$ der nachgewiesenen Bestände ausmacht, ist eine kontinuierliche Koordination über ein online nutzbares Steuerungsinstrument für eine verteilte Digitalisierungsinitiative unabdingbar, um so die maschinelle Zuordnung von Teilen des gesamten Erscheinungsverlaufes zu bestandsführenden Bibliotheken vorzunehmen. Trotz mancher Erfassungsdefizite ist die ZDB dafür das geeignete Instrument.

20 Die auf der Tagung „Presse als historische Quelle“ aufgeflammte Diskussion um die Auswahl in ihrer bedeutungssetzenden Dimension ist wichtig, gilt aber nicht nur für die Auswahl für Digitalisierungen generell, sondern letztlich auch für die (historische) Sammelentscheidung einer Institution. Dem Resümee einer höchstmöglichen Transparenz über solche Auswahlentscheidungen ist unbedingt zuzustimmen, vgl. dazu wie Anm. 5.

21 Es handelt sich um eine unter anderem aus urheberrechtlichen Gründen festgelegte, sicherlich künstliche Trennlinie. 
Eine Annäherung an die hinter den Bestandsdaten stehenden Volumina ist nicht einfach, da Umfänge in der ZDB nicht verzeichnet sind. Eine Hochrechnung aufgrund der Relation der Seitenumfänge zu Titelzahlen im österreichischen ANNO-Portal ${ }^{22}$ und bei den mit Google an der BSB München digitalisierten Zeitungen ergibt einen durchschnittlichen Seitenumfang pro Zeitung von rund 13700 Seiten/Images. Daraus ergäbe sich rein rechnerisch ein geschätzter Gesamtumfang deutscher Zeitungen bis 1945 von 295 Millionen Seiten.

Bislang bereits durchgeführte und laufende Projekte verschiedener regionaler Initiativen, aber auch durch die Europeana angeregter Vorhaben dürften dafür gesorgt haben, dass inzwischen rund 40 Millionen Seiten in digitalisierter Form vorliegen. Definiert man eine kritische Masse in Höhe von rund einem Drittel der deutschen Zeitungsmenge bis 1945 - diese Festlegung hat das Projekt vorgenommen -, ergibt sich daraus eine Zahl von rund 7000 Titeln und rund 98 Millionen Seiten, die digitalisiert werden sollten. Davon sind - s. o. bereits rund 40 Millionen Seiten erreicht, sodass für eine erste große Initiative knapp 60 Millionen Seiten verbleiben.

Bei dieser Dimension ist für eine Kostenabschätzung der Bearbeitungspreis pro Seite der entscheidende Faktor. In den verschiedenen Pilotprojekten zeigte sich allerdings, dass je nach Ausgangsmaterial (sowohl hinsichtlich seiner Physis, seiner Struktur wie auch seines inhaltlichen Gehalts) sehr unterschiedliche Kosten pro Seite angesetzt werden müssen. Dabei spielt der lange Erscheinungszeitraum des Mediums über mehrere Jahrhunderte hinweg eine wichtige Rolle (Herstellungsprozess, Druckverfahren, Gestaltungsnormen). Bedeutsam sind darüber hinaus schon in der Vergangenheit eingeleitete Maßnahmen wie vorliegende Verfilmungen sowie die Anforderungen, die sich aus dem Bedarf einer maschinellen Weiterverarbeitbarkeit des Datenmaterials ergeben.

Im Projekt wurden daher entsprechende Kostenfaktoren ermittelt, deren exakte Bewertung zugleich wesentliche Schritte bei der Vorbereitung einer Digitalisierung bilden.

22 http://anno.onb.ac.at/ (28.11.2017). 
Tab. 1: Im Rahmen des Projekts „Digitalisierung historischer Zeitungen“ ermittelte Kostenfaktoren.

\begin{tabular}{|c|c|}
\hline Ermittelte Kostenfaktoren & Rahmenbedingungen \\
\hline \multicolumn{2}{|l|}{ Aufgabenübergreifend } \\
\hline \multicolumn{2}{|l|}{ Projektleitung und -koordination } \\
\hline \multicolumn{2}{|l|}{ Vorbereitung } \\
\hline \multirow[t]{2}{*}{$\begin{array}{l}\text { Auswahl der zu digitalisierenden Inhalte } \\
\text { und Abgleich mit der ZDB }\end{array}$} & $\begin{array}{l}\text { gegebenenfalls mit wissenschaftlicher Beglei- } \\
\text { tung }\end{array}$ \\
\hline & Skaleneffekte des Mengengerüsts \\
\hline Beschaffung der Vorlagen & $\begin{array}{l}\text { nur Eigenbestand oder gegebenenfalls erhöh- } \\
\text { ter Beschaffungsaufwand aufgrund von } \\
\text { Lückenschluss mit Fremdbestand }\end{array}$ \\
\hline $\begin{array}{l}\text { Prüfung der Vorlagenqualität und Entschei- } \\
\text { dung für eine Vorlagenart (Original vs. Mi- } \\
\text { krofilm) }\end{array}$ & $\begin{array}{l}\text { gewählte Vorlagenart hat Einfluss auf den Kos- } \\
\text { tenrahmen }\end{array}$ \\
\hline \multicolumn{2}{|l|}{$\begin{array}{l}\text { Kollationierung bzw. Prüfung der Vollstän- } \\
\text { digkeit und konservatorischen Eignung }\end{array}$} \\
\hline \multicolumn{2}{|l|}{$\begin{array}{l}\text { Inhouse-Digitalisierung und/oder OCR-Be- } \\
\text { arbeitung/Tiefenerschließung: Prüfung der } \\
\text { Eignung vorhandener Ausrüstung bzw. ge- } \\
\text { gebenenfalls Beschaffung/Aufrüstung von } \\
\text { Scannern und Software }\end{array}$} \\
\hline \multicolumn{2}{|l|}{$\begin{array}{l}\text { Vergabe an Dienstleister (Digitalisierung } \\
\text { und/oder OCR-Bearbeitung/Tiefenerschlie- } \\
\text { Bung): Vorbereitung und Durchführung ei- } \\
\text { nes Vergabeverfahrens }\end{array}$} \\
\hline $\begin{array}{l}\text { Vorbereitung der Materialien: gegebenen- } \\
\text { falls Lückenschluss, konservatorische } \\
\text { Maßnahmen }\end{array}$ & $\begin{array}{l}\text { Aufwand und Ausgestaltung abhängig von den } \\
\text { Projektzielen }\end{array}$ \\
\hline \multicolumn{2}{|l|}{ Workflowplanung und Kostenkalkulation } \\
\hline \multicolumn{2}{|l|}{ Digitalisierung } \\
\hline $\begin{array}{l}\text { Art des Scannereinsatzes und Komplexität } \\
\text { des Digitalisierungsvorganges }\end{array}$ & $\begin{array}{l}\text { abhängig von der physischen Beschaffenheit } \\
\text { der Vorlagen (gebunden/aufgeschnitten; Öff- } \\
\text { nungswinkel; konservatorische Merkmale; Zwi- } \\
\text { schenblätter nach defekten oder fleckigen Sei- } \\
\text { ten) }\end{array}$ \\
\hline Auflösung und Farbtiefe & $\begin{array}{l}\text { Abweichung vom empfohlenen Standard gege- } \\
\text { benenfalls in Abhängigkeit von der Vorlagen- } \\
\text { beschaffenheit }\end{array}$ \\
\hline
\end{tabular}

Qualitätskontrolle (bei Inhouse-Digitalisie-

rung und in Zusammenarbeit mit dem

Dienstleister) 


\begin{tabular}{|c|c|}
\hline Ermittelte Kostenfaktoren & Rahmenbedingungen \\
\hline \multicolumn{2}{|l|}{ Erschließung } \\
\hline \multirow[t]{2}{*}{ bibliografische Erschließung } & $\begin{array}{l}\text { gegebenenfalls jeweils Neuaufnahme/Korrek- } \\
\text { tur für die aufeinander bezogenen Druck- und } \\
\text { Reproduktionsformen erforderlich }\end{array}$ \\
\hline & $\begin{array}{l}\text { RDA sieht für layoutgetreue Digitalisierung ak- } \\
\text { tuell die identische Anlage von Druck- und Re- } \\
\text { produktionskatalogisat vor; hierdurch entste- } \\
\text { hen gegebenenfalls Zusatzaufwände für mögli- } \\
\text { cherweise zahlreiche Titelsplits }\end{array}$ \\
\hline Strukturdatenerschließung & $\begin{array}{l}\text { Aufwand und Grad der Automatisierbarkeit ab- } \\
\text { hängig von der strukturellen Beschaffenheit } \\
\text { der Vorlagen (z. B. Zahl unterschiedlicher Zei- } \\
\text { tungsausgaben, Beilagen, Grad der Einheitlich- } \\
\text { keit der Ausgabenbezeichnungen, Erschei- } \\
\text { nungsfrequenz, wechselnde Kalendersysteme) }\end{array}$ \\
\hline OCR (Antiqua, Fraktur) & $\begin{array}{l}\text { Verarbeitungskosten von Antiqua und Fraktur } \\
\text { unterscheiden sich in der Regel, z. B. durch } \\
\text { pauschale bzw. seitengenaue Errechnung der } \\
\text { Lizenzkosten für die OCR-Software }\end{array}$ \\
\hline Layouterkennung/Artikelseparierung & $\begin{array}{l}\text { abhängig von der strukturellen Beschaffenheit } \\
\text { der Vorlage }\end{array}$ \\
\hline \multicolumn{2}{|l|}{ Qualitätskontrolle } \\
\hline \multicolumn{2}{|l|}{ Bereitstellung } \\
\hline $\begin{array}{l}\text { Einbinden in Präsentationsoberfläche/DFG- } \\
\text { Viewer, gegebenenfalls mit Einrichtung } \\
\text { entsprechender Schnittstellen }\end{array}$ & $\begin{array}{l}\text { abhängig von den jeweiligen Präsentationsvor- } \\
\text { aussetzungen }\end{array}$ \\
\hline Herstellung persistenter Adressierbarkeit & Granularität abhängig von den Projektzielen \\
\hline \multicolumn{2}{|l|}{ Qualitätskontrolle } \\
\hline \multicolumn{2}{|l|}{ Archivierung } \\
\hline Datensicherung & $\begin{array}{l}\text { Auflösung und Farbtiefe der Images haben Ein- } \\
\text { fluss auf den benötigten Speicherplatz }\end{array}$ \\
\hline
\end{tabular}

Je nach Ausgangslage ergeben sich unterschiedliche Kostenkorridore mit einer situationsgebunden weiten Spreizung der Kosten, die zwischen 0,44 Euro (ohne OCR) vom Film und 1,57 Euro (inklusive OCR) für eine Farbdigitalisierung vom Original mit verschiedenen Erschließungstiefen/Weiterverarbeitungsschritten liegen können. Insofern bieten diese Angaben nur eine Orientierung, der Kostenrahmen eines geplanten Projekts ist jeweils individuell anhand des entsprechenden Mengengerüsts zu kalkulieren. Dazu hat das Projekt entsprechende Handreichungen entwickelt. 


\section{Abläufe und Workflow}

Aus den verschiedenen Rahmenbedingungen ergibt sich ein Modellablauf für die Durchführung eines Projekts im Rahmen der Zeitungsdigitalisierung, in dem zwar nicht das exakte Vorgehen und die verwendeten Softwarewerkzeuge (beispielsweise: Welche Workflow-Software wird eingesetzt?) festgelegt sind, aber die Ergebnisse in standardisierter und damit nachnutzbarer Form, sowohl was die Metadaten als auch die Aufbereitung der Digitalisate angeht, erwartet werden.

Um die Vorbereitung eines Projekts in diesem Rahmen möglichst gut zu unterstützen, wurde im Projekt ein Wegweiser entwickelt, der die verschiedenen zu berücksichtigenden Arbeitsschritte benennt und mit den jeweils relevanten Fragestellungen und Entscheidungspunkten aufarbeitet. In diesen online verfügbaren Wegweiser sind praktisch orientierte Handreichungen und Entscheidungshilfen integriert, die sich an den gängigen Abläufen und Fragestellungen bei der Vorbereitung eines Projekts orientieren. ${ }^{23}$ Auch durch diesen Zugangsweg soll sichergestellt werden, dass erforderliche Anpassungen, Korrekturen und Ergänzungen zeitnah vorgenommen werden; die SBB hat sich bereit erklärt, die laufende Pflege der dort enthaltenen und verlinkten Informationen zu übernehmen. ${ }^{24}$

23 Der Wegweiser findet sich unter http://www.zeitschriftendatenbank.de/zeitungsdigitalisierung (28.11.2017).

24 Inhaltliche Hinweise dazu an zdb-hotline@sbb.spk-berlin.de. 
Wegweiser für die Digitalisierung historischer Zeitungen

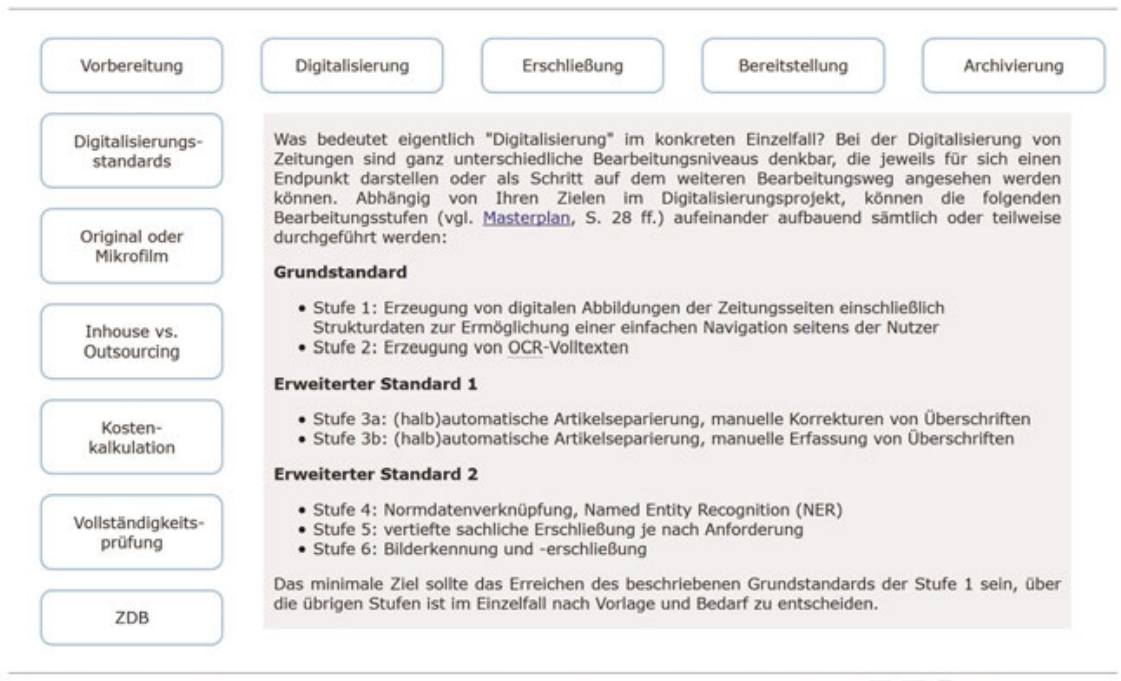

Gefôrdert durch die $\mathbf{D F}$

Abb. 1: Wegweiser, der bei der Vorbereitung eines Projekts zur Digitalisierung historischer Zeitungen zum Einsatz kommen soll.

\section{Perspektive Nationales Zeitungsportal}

Alle genannten Anstrengungen kulminieren in dem Angebot eines entsprechenden nationalen Zugangsfensters für Zeitungen, das den Zeitungsportalen anderer Länder vergleichbar komfortable Überblicke und Sucheinstiege bietet. Dabei ist von vornherein auch eine Datenschnittstelle zu berücksichtigen. Im Projekt bestand Konsens, dass dieser Zugang ähnlich der Realisierung des Archivportal-D innerhalb der DDB errichtet werden sollte. Wesentliche zusätzliche Funktionen über das in diesem Kontext bereits Vorhandene hinaus sind eine übergreifende Volltextsuche in den digitalisierten Zeitungsbeständen, zusätzliche Einstiegspunkte über Zeitungstitel, Kalender, Erscheinungsorte, Verbreitungsgebiete, die Integration des Zeitungsviewers (DFG-Viewer) in die Portalumgebung mit stufenloser Zoom-Funktion, mit Highlighting von Suchtreffern und der Möglichkeit, Textabschnitte im Volltext per Copy-and-paste direkt zu kopieren und weiterzuverwenden sowie schließlich die Nachnutzung der konsolidierten Daten- und Präsentationsstrukturen der ZDB. Hinzu kommt eine konsistente 
Möglichkeit, Zeitungen bzw. Einzelausgaben persistent zu referenzieren und sie somit zitierfähig zu machen. Wesentliches konstitutives Prinzip bei der Implementierung wird dabei der Verbleib der Image-Daten bei den jeweiligen Urhebern sein, auch die Volltexterzeugung ist den Partnern überlassen. Diese verteilte Datenhaltung entspricht so den eingeübten Usancen der Infrastruktur. Zugleich ist klar, dass eine leistungsfähige Volltextsuche auf der Basis eines zentral vorgehaltenen Index entstehen sollte, zumal, wenn Anreicherungen der Daten erfolgen sollen. Berücksichtigt werden muss perspektivisch ebenfalls, dass es Einrichtungen geben wird, die aus unterschiedlichen Gründen eine gesicherte, auf Dauer angelegte Vorhaltung und Bereitstellung der Bilddaten nicht selbst übernehmen wollen. Das Portal wird dabei auf das (auch im EU-Projekt „Europeana Newspapers“ verwendete) International Image Interoperability Framework (IIIF) setzen, um an verschiedenen Orten gehostete Daten in einer einheitlichen gemeinsamen Präsentation zusammenzuführen. ${ }^{25}$

Dem empfehlenden Vorschlag des Projektkonsortiums an die DDB, ein Nationales Zeitungsportal aufzubauen, ist diese in einem ersten Schritt inzwischen gefolgt: Ein entsprechender Antrag an die DFG ist gestellt.

\section{Ausblick}

Das Projekt hat mit seinen Ergebnissen die Eckpunkte und Rahmenbedingungen für den systematischen und breiten Einstieg in die Digitalisierung historischer Zeitungen aufgezeigt. Die Phasierung einzelner Etappen unter Ausbalancierung der fördertechnischen, finanziellen und organisatorischen Möglichkeiten der DFG bildet nun den nächsten Schritt und wird Gegenstand der angekündigten Förderinitiative im Frühjahr 2018 sein. Ob der Fokus zunächst auf einem Einstieg bei großen bestandshaltenden Einrichtungen liegt, wie es Alexander Rindfleisch bereits 2010 vorgeschlagen hat, wird sich zeigen. ${ }^{26}$

Es entstanden und entstehen über die im Projekt realisierten Module hinaus weitere Bausteine für eine verteilte Infrastruktur. Ein wesentliches Element ist

25 http://iiif.io/ (3.12.2017).

26 In dieser Arbeit schlug Rindfleisch ein nationales Zeitungsdigitalisierungsprogramm vor, das in drei Stufen erfolgen sollte: zentraler Start auf Basis der Daten großer Bibliotheken und weitgehender Berücksichtigung der Bestände des Mikrofilmarchivs (Auswahl durch Expertengremium, Finanzierung über die DFG), eine zweite dezentral, landesfinanzierte Stufe, die sich inhaltlich eng an das zentrale Programm anlehnt, und schließlich eine dritte Phase als DFG-Aktionslinie. Vgl. Rindfleisch, Alexander: Stand und Perspektive der Zeitungsdigitalisierung im internationalen Vergleich. Berlin: Institut für Bibliotheks- und Informationswissenschaft der Humboldt-Universität 2010. doi.org/10.18452/ (3.12.2017). 
dabei das Nationale Zeitungsportal, in dem die Bestände in den deutschen Bibliotheken den Ausgangspunkt bilden, was aber letztlich in weitere Kooperationen einmünden muss: Neben der Präsentationsschicht wird daher die Datenschnittstelle große Bedeutung haben, wenn es darum geht, den deutschen Sprachraum mit seinen vielfachen Bezügen gesamtheitlich zu integrieren und für vergleichende Analysen in Kooperationen mit anderen Partnern zu öffnen.

Auch für die ZDB als Steuerungsinstrument für die Digitalisierungsaktivitäten und die sie tragenden Bibliotheken zeichnen sich weitere Aufgaben ab: Neben bereits in Eigenleistung erbrachten Ergänzungen von Geokoordinaten zu Orten generell und der Ausweitung der Informationen zu Verbreitungsorten bestehen diese insbesondere in der verbesserten Erschließung (normierte Erscheinungsjahre, standardisierte Erfassung von Information und Pflege von Digitalisierungsabsichten). Ein weiterer wichtiger Beitrag ist in der Integration der Daten des Mikrofilmarchivs (MFA) zu sehen, da so auch die verfilmten Bestände unter einer Oberfläche zugänglich und sichtbar gemacht würden - eine unerlässliche Information für die Steuerung von Digitalisierungsprojekten. Zugleich würden damit Bestände aus Archiven nachgeführt, ein Teilschritt zu einer besseren Sichtbarkeit der Zeitungsbestände in Archiven insgesamt.

Der Bedarf an einer verbesserten Nachverarbeitung entstehender Images korrespondiert mit den Aufbauaktivitäten des DFG-Projekts OCR-D. Das Spektrum der hier erkennbaren Themenfelder reicht vermutlich über die bereits begonnenen Maßnahmen hinaus und erstreckt sich auch auf die forschungsnahe Weiterentwicklung von Verfahren. Bislang ermittelte Bedarfsstellungen für die Arbeit mit digitalen Korpora aus Daten und Bildern umfassen - neben der weiteren Optimierung und rekursiven Nachführung von OCR-Verfahren - z. B. die Erkennung von grafischen Elementen zur Trennung von Artikeln (Separatoren) und Separierungsverfahren, die Erkennung von Tabellen, die Strukturerkennung sowie die Bilderkennung und -extraktion. Aus Nutzeranalysen ist bekannt, dass die Suchanfragen im Zeitungskontext zu hohen Prozentsätzen Personen oder Ortsnamen gelten; demzufolge kommt der (halb-)automatischen Erkennung von Entitäten eine besondere Bedeutung zu. Erste Erfahrungen zur Unterscheidung von Namen liegen vor, diese müssen allerdings auf Ortsnamen ausgedehnt werden. Dabei sind die insbesondere bei Namen zahllosen Schreibvarianten zu berücksichtigen, die aber ebenso historisch in Zeitungen regionale bis individuelle Spezifika betreffen können. Insofern ist es dringend zu wünschen, dass im Bereich technikgetriebener Anreicherungsverfahren spätestens dann, wenn die Menge des zur Verfügung stehenden und initial mit OCR aufbereiteten Materials durch die Digitalisierung steigt, Fördermöglichkeiten zur Wei- 
terentwicklung automatischer Erschließungs- und Anreicherungsverfahren bestehen.

In einer aus verschiedenen Komponenten bestehenden verteilten Infrastruktur kann der Medientyp Zeitung einen paradigmatischen Status erhalten. Die einzelnen Module dienen nämlich nicht nur diesem einen Medientyp, für den also keine gesonderte Infrastruktur aufgebaut wird, sondern stehen in anderen Kontexten zugleich ganz anderen Anforderungen zur Verfügung. Dies gilt sowohl für die Workflows der Digitalisierung selbst, die technischen Parameter, die technischen Werkzeuge und Verfahren, die OCR und weitere Datenanreicherungs- und Extraktionsverfahren als auch für die DDB, deren Grundmuster übernommen werden, oder für die ZDB. Im Gegenteil, der Impuls zur Weiterentwicklung der Präsentationsfläche für Zeitungen hat letztlich die fast vollzogene Ablösung der alten OPAC-Strukturen der ZDB beschleunigt.

Zum heutigen Zeitpunkt kann konstatiert werden, dass in die Digitalisierung von historischen Zeitungen in Deutschland und ihre Förderung Bewegung gekommen ist. Auch wenn in der Vergangenheit eine Reihe verdienstvoller regionaler Initiativen den Bestand digitalisierter Zeitungen erhöht hat, bedarf es doch einer zentralen Initiative, um die im internationalen Vergleich bescheidene Situation der Digitalisierung historischer Zeitungen im „Zeitungsland Deutschland“ zu verändern. Dass dies in Gang gekommen ist und voraussichtlich deutlich über eine bloße Bereitstellung von Digitalisaten hinausgeht, ist zu einem erheblichen Maß das Verdienst von Thomas Bürger. 